\title{
The Evaluation of Esophageal Motility Using Infusion Manometry with a Trans-nasal Endoscope: A Preliminary Study
}

\author{
Kosaku Hata ${ }^{1}$, Masanori Ito ${ }^{1}$, Tomoyuki Boda ${ }^{2}$, Takahiro Kotachi ${ }^{2}$, Mariko Kiso ${ }^{1}$, \\ Yuichi Hiyama ${ }^{1}$, Kazutaka Kuroki ${ }^{1}$, Naoki Yorita ${ }^{1}$, Naoko Nagasaki ${ }^{1}$, Madina Abduwaili ${ }^{1}$, \\ Shinji Tanaka ${ }^{2}$ and Kazuaki Chayama ${ }^{1}$
}

\begin{abstract}
:
Objective The Chicago classification using high-resolution manometry (HRM) has been proposed for the diagnosis of esophageal motility disorders. However, HRM is expensive, and few patients are likely to benefit from this method. We established a method using a raised infusion catheter called "esophageal manometry using a trans-nasal endoscope". In this study, we examined the clinical utility of this new method for the assessment of patients with dysphagia.

Methods We enrolled 124 consecutive patients who complained of dysphagia and were suspected of having esophageal motility disorders. Patients underwent manometry using a trans-nasal endoscope at Hiroshima University Hospital from September 2008 to December 2018. An esophageal biopsy was performed during the procedure from the thoracic esophagus to detect eosinophilic esophagitis. In addition, we examined the clinical courses of patients diagnosed with achalasia.

Results The diagnostic ability of manometry using a trans-nasal endoscope was $98 \%$, and no major complications associated with the procedure were recorded. About $30 \%$ of patients were diagnosed with achalasia, and most $(77 \%)$ were well controlled by medications or balloon dilation. One patient was diagnosed with eosinophilic esophagitis.

Conclusion Manometry using a trans-nasal endoscope is beneficial and can be used for the clinical management of patients with dysphagia.
\end{abstract}

Key words: esophageal manometry, endoscope, achalasia

(Intern Med 59: 887-890, 2020)

(DOI: 10.2169/internalmedicine.3734-19)

\section{Introduction}

During swallowing, the bolus of food is transported into the stomach from the mouth by the movement of the esophagus (1-3). Esophageal motility disorders are conditions in which this esophageal movement is impaired for some reason, leading to problems with swallowing. In clinical practice, patients with esophageal motility disorders experience a markedly reduced quality of life, although the disorders are not considered to be malignant.

Esophageal manometry is essential for the diagnosis of esophageal motility disorders. Thus far, the infused catheter and solid-state sleeve sensor methods have been used for the evaluation of esophageal motility, both of which involve blind insertion. Recently, the Chicago classification was developed for the international standardization of esophageal motility disorders. In this classification system, highresolution manometry (HRM) is proposed for diagnostic purposes $(4,5)$. However, HRM systems involving a solid-

${ }^{1}$ Department of Medicine and Molecular Science, Hiroshima University, Japan and ${ }^{2}$ Department of Endoscopy, Hiroshima University Hospital, Japan

Received: July 29, 2019; Accepted: October 24, 2019; Advance Publication by J-STAGE: December 6, 2019

Correspondence to Dr. Masanori Ito, maito@hiroshima-u.ac.jp 
state sensor are expensive, and the number of patients who actually require HRM is quite limited in clinical practice (6). Another problem lies in the discomfort and risk associated with esophageal motility testing due to the blind insertion of trans-nasal catheters.

Recently, trans-nasal endoscopes have gained worldwide popularity. These devices can help reduce the discomfort and risks mentioned above (7). Kawai et al. first reported a novel infusion catheter method using trans-nasal endoscopy (8). Along similar lines, we have been performing esophageal manometry using trans-nasal endoscopy (MTNE) and newly developed catheters since 2008.

We herein report the clinical utility of M-TNE for evaluating the cause of dysphagia based on our clinical experience. In addition, we report the results of an esophageal biopsy to diagnose eosinophilic esophagitis $(\mathrm{EoE})$ in these patients. Finally, we describe the prevalence of achalasia in the cohort and describe the clinical courses of these patients.

\section{Materials and Methods}

\section{Patients}

We enrolled 124 consecutive patients (60 men and 64 women; average 57.5 years old) who complained of dysphagia or esophageal obstruction and were suspected of

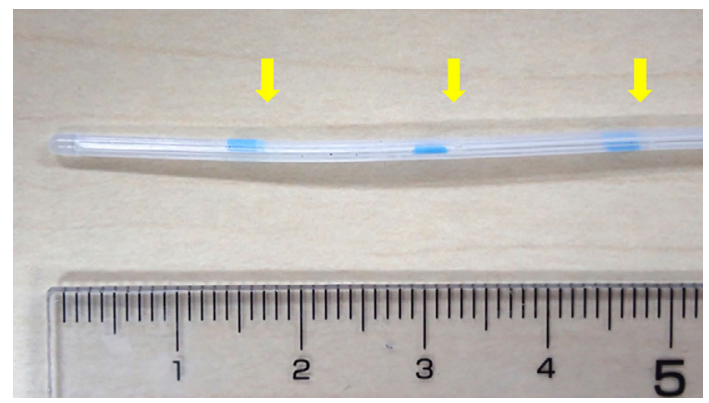

Figure 1. Originally developed catheter with 3 side holes (yellow arrows) at $1.5-\mathrm{cm}$ intervals. having esophageal motility disorders. All 124 patients underwent M-TNE at Hiroshima University Hospital from September 2008 to December 2018. We excluded patients with esophageal tumors or severe reflux esophagitis (Los Angeles grade $\mathrm{C} / \mathrm{D})$. We retrospectively examined the clinical courses of patients diagnosed with achalasia. The diagnosis of achalasia was made comprehensively based on the results of MTNE, esophagography and endoscopic findings. The ethics committee at Hiroshima University approved the protocol of this study.

\section{Trans-nasal endoscopy and infusion manometry}

First, patients received lidocaine spray to the nostrils and pharynx. A nasal pretreatment catheter coated with lidocaine gel was then inserted gently into the nostril, which allowed the patient to breathe more easily. After 3 minutes, the catheter was removed, and an esophagoduodenogastroscope (GIF N260; Olympus, Tokyo, Japan) was inserted transnasally. After routine observation, we inserted a catheter through the forceps hole and evaluated the lower esophageal sphincter (LES) pressure and the frequency of primary peristaltic waves with dry and wet swallowing while the distal side hole was visually confirmed to be through the LES. We used an originally developed catheter with 3 side holes at 1.5-cm intervals (Fig. 1). The patient was in the left lateral decubitus position during the examination, and the inspection time was about 20 minutes.

The classification of esophageal motility disorders was the same as that described in previous studies $(9,10)$. The evaluation based on the Chicago classification was not adopted in the present study because we were unable to use HRM at the same time. We diagnosed achalasia based on the loss of the primary peristalsis wave, failure of the LES, and endoscopic findings. Representative results of a patient with achalasia are shown in Fig. 2.

During the procedure, we performed esophageal biopsies (two sites) from the thoracic esophagus to diagnose the presence of EoE. If we detected $>20$ eosinophils in the magnified field, we diagnosed the patient as having EoE (11).

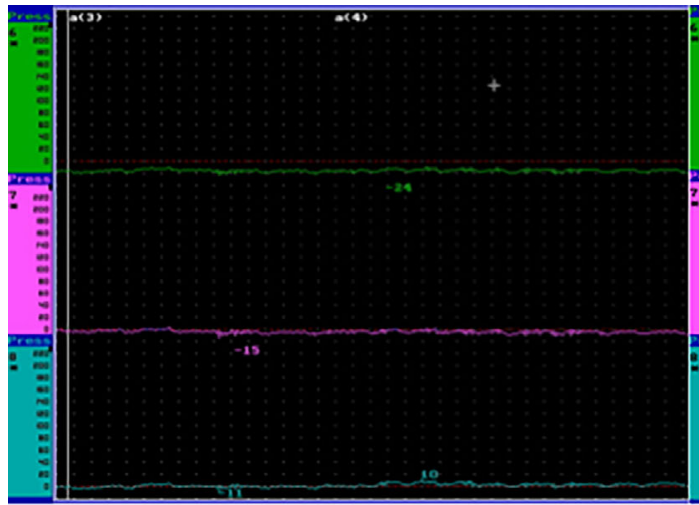

A

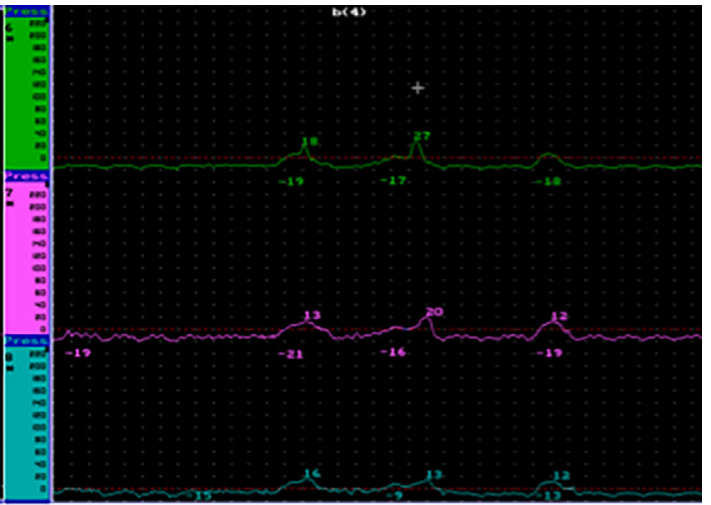

B

Figure 2. Manometry using a trans-nasal endoscope in a patient with achalasia. (A) Loss of primary peristalsis wave, (B) simultaneous waves. 
Table 1. Clinical Diagnosis of 124 Patients with Esophageal Manometry Using Trans-nasal Endoscopy Methods.

\begin{tabular}{lcc}
\hline Achalasia & 35 & $(28.2)$ \\
Other esophageal motility disorders* & 42 & $(33.9)$ \\
Secondary esophageal motility disorders & 4 & $(3.2)$ \\
No abnormality & 41 & $(33.1)$ \\
Undiagnosed & 2 & $(1.6)$ \\
\hline & & $\mathrm{n}(\%)$ \\
\hline
\end{tabular}

*Diffuse esophageal spasm (17), Nutcracker esophagus (7), Nonspecific esophageal motility disorders (18)

We judged the effect of each treatment for achalasia using the Eckardt score, which is the sum of the achalasia symptom scores for dysphagia, regurgitation, chest pain, and weight loss and used to assess achalasia symptom severity and treatment effectiveness. An Eckardt score of $<3$ points was considered to indicate improvement of the disease (12).

\section{Statistical analyses}

Categorical data were compared using Fisher's exact test, the chi-square test, or the Mann-Whitney $U$-test, as appropriate. We considered $\mathrm{p}$ values of $<0.05$ to represent statistical significance. The JMP 12.1 statistical software program (SAS Institute, Cary, USA) was used for all calculations.

\section{Results}

\section{The clinical diagnosis of 124 patients using M-TNE}

The evaluation of esophageal motility by M-TNE was possible in 122 of 124 (98.4\%) patients. In two cases, we were unable to regulate the patient's severe coughing reaction, which made a diagnosis impossible. No adverse events occurred in the remaining patients.

The final diagnoses are summarized in Table 1. Thirtyfive $(28.2 \%)$ patients were diagnosed with achalasia. Besides achalasia, other esophageal motility disorders were found in $42(33.9 \%)$ patients (17 cases of diffuse esophageal spasm, 7 of nutcracker esophagus, and 18 of nonspecific esophageal motility disorders). Secondary disorders were detected in four patients. Of the four patients, three had scleroderma, and one had esophageal diverticulum. In contrast, 41 (33.1\%) patients were not diagnosed with any disorder.

\section{Clinical course of patients diagnosed with achalasia}

We retrospectively examined the clinical courses of patients diagnosed with achalasia. After the diagnosis of achalasia, patients received medication (calcium antagonists or nitrates) for four weeks as first-line therapy. Balloon dilation to $30 \mathrm{~mm}$ with an achalasia balloon (Boston Scientific Corporation, Marlborough, USA) was performed in patients refractory to this intervention. For patients who remained refractory after balloon dilation, we performed surgery (Heller-Dor) or per-oral endoscopic myotomy (POEM).
Table 2. Treatment for Achalasia of 35 Patients.

\begin{tabular}{lcc}
\hline Drug medication & 16 & $(45.7)$ \\
Endoscopic balloon dilation & 11 & $(31.4)$ \\
Per-oral endoscopic myotomy & 3 & $(8.6)$ \\
Surgery & 4 & $(11.4)$ \\
Follow-up & 1 & $(2.9)$ \\
\hline & & $\mathrm{n}(\%)$ \\
\hline
\end{tabular}

The final results are presented in Table 2. Medication was effective in $16(45.7 \%)$ patients, and endoscopic balloon dilation was effective in $11(31.4 \%)$ patients. POEM or surgery was carried out in $7(20.0 \%)$ patients. The median Eckardt scores pre-/post-treatment were $4 / 1$ by drug medication, $6 / 1$ by endoscopic balloon dilation, $4 / 0$ by POEM, and $7.5 / 1.5$ by surgery, respectively.

\section{Prevalence of EoE}

All 124 patients received an esophageal biopsy and histological examination. One patient was diagnosed histologically with EoE. We observed no adverse events caused by the esophageal biopsy. The $95 \%$ confidence interval for the prevalence of EoE was 0.14-4.4\%.

\section{Discussion}

In the present study, we demonstrated the clinical utility of M-TNE in patients with dysphagia. TNE is becoming popular worldwide, particularly for cancer screening (13). Using a trans-nasal endoscope, the catheter can be inserted under direct vision, resulting in smooth and safe insertion. Furthermore, it is possible to evaluate the esophageal motility in most patients using this approach, suggesting that this method is easy, safe, and useful. This method can be used more generally to diagnose diseases of the esophagus, such as esophageal cancer, herniation, diverticulum, and so on.

We modified an originally developed catheter by incorporating 3 side holes at $1.5-\mathrm{cm}$ intervals. Wider intervals may be beneficial for the detection of peristalsis in the esophagus. However, if the holes are spaced too widely, the most distant one might not be recognized. The $1.5-\mathrm{cm}$ intervals used in our catheter appear to be optimal for M-TNE. In addition, esophageal peristalsis was able to be observed directly, further helping in the diagnosis of esophageal dysmotility.

Another merit of M-TNE lies in the ability to perform a simultaneous histological examination by taking an esophageal biopsy specimen. EoE is a representative disease that causes esophageal symptoms, including dysphagia. EoE is increasingly common in Japan, and it is important that this disease be accurately diagnosed using both an endoscopic examination and an esophageal biopsy (14). In the present study, we routinely recovered two biopsy specimens from the esophagus. Despite this, only one patient was diagnosed with EoE. This suggests that the prevalence of EoE in pa- 
tients with dysphagia, at least in this cohort, was not high. However, whether or not an esophageal biopsy via TNE is sufficient should be discussed, as the biopsy forceps are small compared with those normally used for this purpose, and the number of biopsy specimens was limited.

At present, the diagnosis of achalasia is based on the Chicago classification using HRM (2). Recently, a more detailed classification system was proposed for the diagnosis and management of patients with achalasia (14). Achalasia was diagnosed in $28.2 \%$ of patients in the present study, which is comparable to the results of a previous report (10). M-TNE may be sufficient for the primary diagnosis of achalasia. In the present study, $45.7 \%$ of patients reacted well to calcium channel blockers for first-line therapy. Second-line (balloon dilation) or third-line (POEM or surgery) therapy was necessary for non-responders. A precise diagnosis may be needed in patients with a poor response in order to determine the most appropriate medications. In the future, POEM may become the first-line therapy for achalasia, and HRM may become essential for determining the ideal approach for the POEM procedure (15).

The main issue with M-TNE is the difficulty of evaluating the LES pressure. Whereas we set the top hole on the LES position with direct viewing, respiratory movement may interrupt the evaluation of LES pressure, necessitating more time to obtain a reading. Some improvements may therefore be necessary in the future, such as the incorporation of a sleeve assembly on the top of the catheter, as previously reported (16).

Several limitations associated with the present study warrant mention. First, it has not been evaluated with M-TNE after treatment. In the next step, we are planning to reevaluate the M-TNE findings after treatment and discuss the differences observed. Second, since M-TNE can evaluate the motility only in the lower esophagus, it may be difficult to diagnose some patients with esophagogastric junction outflow obstruction, diffuse esophageal spasm, nutcracker esophagus, or achalasia type III. In these patients, an additional examination by HRM may be essential. M-TNE is a simple but not a definite method for correct diagnosis of esophageal motility disorder. Although the diagnostic ability of M-TNE should be determined by further studies, M-TNE may have potential utility for primary screening of patients with dysphagia, considering the limited availability of HRM in Japan. For the further evaluation of the clinical utility of M-TNE, a direct comparison between M-TNE and HRM should be performed as the next step.

In conclusion, M-TNE may be useful for the clinical management of patients with dysphagia as a primary examination.
The authors state that they have no Conflict of Interest (COI).

\section{References}

1. Leopold. NA. Swallowing, ingestion and dysphagia: a reappraisal. Arch Phys Med Rehabil 64: 371-373, 1983.

2. Logemann. JA. Evaluation and Treatment of Swallowing Disorders. 2nd ed. PRO-ED, Austin, Texas, 1998.

3. Feinberg. MJ. Radiographic techniques and interpretation of abnormal swallowing in adults and elderly patients. Dysphagia 8: 356-358, 1993

4. Kahrilas PJ, Bredenoord AJ, Fox M, et al. The Chicago classification of esophageal motility disorders, v3.0. Neurogastroenterol Motil 27: 160-174, 2015.

5. Bredenoord AJ, Fox M, Kahrilas PJ, et al. Chicago classification criteria of esophageal motility disorders defined in high resolution esophageal pressure topography. Neurogastroenterol Motil 24 (Suppl 1): 57-65, 2012.

6. Kuribayashi S, Hosaka H, Kawada A, et al. Esophageal motility disorders (except GERD). Journal of Japanese Society of Gastroenterology 111: 1933-1939, 2014.

7. Kawai T, Miyazaki I, Yagi K, et al. Comparison of the effects on cardiopulmonary function of ultrathin transnasal versus normal diameter transoral esophagogastroduodenoscopy in Japan. Hepatogastroenterology 54: 770-774, 2007.

8. Kawai T, Yamaguchi T, Yagi K, et al. Impact of transnasal ultrathin esophagogastroduodenoscopy (UT-EGD) in the evaluation of esophageal peristaltic function. J Gastroenterol Hepatol 23 (Suppl 2): 181-185, 2008.

9. Ouyang A, Cohen S. Motility disorders of the esophagus. In: Bockus Gastroenterology. 5th ed. Haubrich W, Schafener F, Berk JE, Eds. WB Saunders, Philadelphia, 1995: 418-436.

10. Kusano M, Maeda M, Shimoyama Y, Kuribayashi S, Mori M. Esophageal motor function in nonachalasia motility disorders. Nihon Shokakibyo Gakkaishi 100: 1095-1105, 2003 (in Japanese).

11. Kinoshita Y, Furuta K, Ishimaura N, et al. Clinical characteristics of Japanese patients with eosinophilic esophagitis and eosinophilic gastroenteritis. J Gastroenterol 48: 333-339, 2013.

12. Eckardt VF. Clinical presentations and complications of achalasia. Gastrointest Endosc Clin North AM 11: 281-292, vi, 2001.

13. Kawai T, Yanagizawa K, Naito $S$, et al. Evaluation of gastric cancer diagnosis using new ultrathin transnasal endoscopy with narrow-band imaging: preliminary study. J Gastroenterol Hepatol 29 (Suppl 4): 33-36, 2014

14. Ihara E, Muta K, Fukaura K, Nakamura K. Diagnosis and treatment strategy of achalasia subtypes and esophagogastric junction outflow obstruction based on high-resolution manometry. Digestion 95: 29-35, 2017.

15. Tuason J, Inoue H. Current status of achalasia management: a review on diagnosis and treatment. J Gastroenterol 52: 401-406, 2017.

16. Hananoki M, Haruma K, Tsuga K, et al. Evaluation of lower oesophageal sphincter pressure using endoscopic manometric sleeve assembly. J Gastroenterol Hepatol 15: 121-126, 2000.

The Internal Medicine is an Open Access journal distributed under the Creative Commons Attribution-NonCommercial-NoDerivatives 4.0 International License. To view the details of this license, please visit (https://creativecommons.org/licenses/ by-nc-nd/4.0/).

(C) 2020 The Japanese Society of Internal Medicine Intern Med 59: 887-890, 2020 